DOI: $10.17234 / S R A Z .65 .9$

UDK: 81'255.4-05 Meschonnic, $\mathrm{H}$. UDK: 81'255.4-05 Guberina, P.

Original scientific paper

Reçu le 7 juin 2020

Accepté pour la publication le 25 novembre 2020

\title{
Sur Henri Meschonnic, Petar Guberina et le rythme dans la traduction
}

Vanda Mikšić

Université de Zadar

vmiksic@unizd.hr

Lors du processus traductif, il est inévitable pour chaque traducteur littéraire de se poser la question du rythme, qui participe à la construction du texte dans sa singularité et véhicule des effets poétiques. Dans la présente contribution, nous nous sommes penchée sur la réflexion traductologique autour de ce concept, en mettant en parallèle deux théoriciens d'envergure du XXe siècle, le stylisticien croate Petar Guberina et le poéticien français Henri Meschonnic. Dans la partie conclusive, nous avons pu, malgré leurs positions théoriques distinctes, mettre en évidence quelques points convergents, liés notamment à leur conception du texte, de la parole $(\mathrm{du}$ corps, de la voix) et de l'oralité, du rôle que le rythme revêt dans chaque œuvre littéraire, et de la nécessité de l'intégrer dans le processus traductif. Ces convergences constituent, à notre avis, des repères théoriques précieux pour la pratique traductive littéraire d'aujourd'hui.

Mots-clés : Guberina, Meschonnic, rythme, oralité, traduction

\section{Pourquoi rapprocher Henri Meschonnic et Petar Guberina autour de la notion du rythme?}

La question que nous nous posons en ouverture à la présente contribution ne peut se contenter d'une réponse unique. Loin de vouloir simplement marquer, avec un sujet de circonstance, le premier centenaire des études romanes à la Faculté de Philosophie et Lettres de l'Université de Zagreb, en faisant un hommage, plus que mérité d'ailleurs, à Petar Guberina, l'un des professeurs du Département d'études romanes, dont il assurait la direction dans les années 1950, notre intention est avant tout de rapprocher deux linguistes du XXe siècle, deux théoriciens à la renommée mondiale qui se sont penchés à la fois sur les questions du rythme et de la traduction, voire sur la question du rythme dans le processus traductif. Malgré les différences entre leurs optiques et leurs terminologies, leurs conclusions convergent sur plusieurs points essentiels que nous examinerons ici et qui peuvent s'avérer profitables pour la théorie et la pratique de la traduction littéraire d'aujourd'hui. 
Rappelons rapidement que Henri Meschonnic (1932 - 2009), linguiste français, professeur universitaire, poéticien de la traduction, poète et traducteur (de la Bible, notamment), a mis en évidence la place du rythme dans le texte (littéraire) en élaborant, à partir des années 1970, sa poétique du traduire - désormais célèbre - autour des concepts du rythme, de l'historicité, de l'oralité et de la subjectivité. ${ }^{1}$ Son approche poétique se situe en prolongement de la réflexion d’Émile Benveniste.

Pour Petar Guberina (1913 - 2005), linguiste (phonéticien) croate, professeur universitaire, théoricien de la stylistique, imprégné de la culture et de la théorie linguistique francophones, c'est surtout la réflexion de Charles Bally dans son Traité de stylistique française (1909) qui représentait le point de départ du développement de ses idées. Dès la rédaction de sa thèse de doctorat (Sorbonne, 1939), il s'est penché sur la langue parlée : auteur de la théorie verbo-tonale de la Parole et de l'Audition, il a conçu, avec Paul Rivenc, l'approche théorique structuro-globale audiovisuelle (SGAV) de l'enseignement des langues. Ses recherches stylistiques, combinées avec l'enseignement universitaire, ont abouti à un manuel universitaire intitulé Stilistika (La Stylistique, 1967)², comportant deux chapitres consacrés justement aux questions du rythme et de la traduction. ${ }^{3}$ Par ailleurs, il s'est intéressé à la poésie et aux cultures africaines, grâce notamment à son amitié avec Aimé Césaire.

Quant à la notion du rythme, souvenons-nous que tout au long de l'histoire elle fut développée dans deux directions principales, dont les sources remontent à l'ancienne Grèce. La première fut proposée par Héraclite (VIe-Ve s.), et la seconde par Platon (Ve-IVe s.). Le mot même provient de l'ancien grec : le substantif rhuthmòs signifiait la manière de fluer, et venait à son tour du verbe rheo. Héraclite voyait dans le rythme une dynamique, un mouvement, une progression, impliquant une continuité, une évolution. Selon lui, il était impossible d'établir un modèle qui se répète. Platon, en revanche, définissait le rythme comme une alternance du plein et du vide, du régulier et de l'irrégulier, de l'accentué et de l'inaccentué. Il s'agit bien d'une conception binaire, métrique du rythme, englobant la répétition du même modèle (sonore) et la régularité (le battement du cœur, le train, l'alternance des saisons), impliquant donc la mesure et les

1 Parmi ses ouvrages consacrés au rythme nous pouvons citer : Pour la poétique II. Epistémologie de l'écriture, poétique de la traduction (1973); Le signe et le poème (1975); Critique du rythme. Anthropologie historique du langage (1982; 2009); Politique du rythme, politique du sujet (1995); Traité du rythme, des vers et des proses, avec Gérard Dessons (1998); Poétique du traduire (1999).

2 La 2e édition de l'ouvrage a été publiée intégralement en 2016 sur le site du Département d'études slaves de la Faculté de Philosophie et Lettres de l'Université de Zagreb (https://stilistika.org/petar-guberina-stilistika). Toutes nos citations de cet ouvrage dans la suite de ce texte proviennent de cette édition ; l'ouvrage n'étant pas paginé, nous ne pouvons qu'apporter le numéro de la partie et du chapitre où la citation donnée apparaît, par ex. (2016: 1, III - première partie, troisième chapitre).

3 Un autre ouvrage qu'il y a lieu de mentionner ici est Povezanost jezičnih elemenata (Interdépendance des éléments linguistiques), datant de 1952. 
proportions, mais aussi une discontinuité interne. Il va sans dire que la conception du rythme platonicienne était historiquement dominante en Occident.

\section{Le rythme, et la traduction du rythme, dans la réflexion d'Henri Meschonnic}

Avant d'aborder la théorie du rythme d'Henri Meschonnic, il nous faut présenter brièvement les idées de base de sa réflexion qui se veut poétique. En fait, il considère la pensée poétique comme la manière particulière dont un sujet transforme « les modes de signifier, [...], de vivre dans le langage. C'est un mode d'action sur le langage. » (1999 : 30) Contre le binarisme, le dualisme structuraliste (forme/contenu, son/sens) et la dichotomie du signe (signifiant/ signifié), Meschonnic prône la continuité. Dans cette perspective, il insiste sur le discours en tant qu'unité langagière : « [...] l'unité du langage n'est pas le mot, et ne peut donc pas être le sens, son sens. [...] Mais l'unité est le discours. Le système du discours. » (id. : 23) Tout discours, d'après Meschonnic, « suppose le sujet, inscrit prosodiquement, rythmiquement dans le langage, son oralité, sa physique » (id. : 74). L'écriture n'est pas séparée de la voix, donc du corps - c'est le corps qui s'exprime dans le texte à travers les marques de l'oralité : «L'oral est compris comme un primat du rythme et de la prosodie dans l'énonciation. » (id. : 117) Dans un ouvrage antérieur, Meschonnic élabore le concept de l'oralité :

L'oralité est le rapport nécessaire, dans un discours, du primat rythmique et prosodique de son mode de signifier à ce que dit ce discours. L'oralité est collectivité et historicité. [...] L'oralité apparaît le mieux dans ces textes portés d'abord par une tradition orale, avant d'être écrits : la Bible en hébreu, ou Homère, les textes africains, toute littérature 'populaire'. (2009 [1982] : 280)

Chaque auteur propose une prosodie et une sémantique personnelles, de sorte que dans un texte (littéraire) l'oralité se trouve intensifiée.

Henri Meschonnic définit le rythme langagier comme

l'organisation des marques par lesquelles les signifiants, linguistiques et extra-linguistiques [...] produisent une sémantique spécifique, distincte $d u$ sens lexical, et que j'appelle la signifiance : c'est-à-dire les valeurs propres à un discours et à un seul. Ces marques peuvent se situer à tous les 'niveaux' du langage : accentuelles, prosodiques, lexicales, syntaxiques. (id. : 216-217)

Le rythme est pour lui une «synthèse de tous les éléments du discours, y compris la situation, l'émetteur, le récepteur. » (id. : 223) Nous pouvons dire qu'il s'écarte explicitement de la position platonicienne, en considérant le rythme comme le principe d'organisation du discours, ou selon ses propres mots, « l'organisation du mouvement de la parole dans l'écriture » (1995b : 515). En d'autres termes, le rythme, c'est la subjectivité qui transfère l'historicité à la forme-sens.

Quand il s'agit de la traduction, Meschonnic considère qu'elle n'est pas un simple instrument de communication interlinguistique et interculturelle, mais 
« le meilleur poste d'observation sur les stratégies de langage » (1999: 14). Pour lui, il ne suffit pas de traduire le sens, il ne suffit pas de rendre la forme (il faut dépasser cette dichotomie structuraliste) : le rythme constitue une valeur de la traduction, un critère de son historicité, et de sa poéticité. Dans ses ouvrages, Meschonnic s'obstine à répéter que la traduction d'un texte (littéraire) doit «faire ce que fait le texte littéraire, par sa prosodie, par son rythme, sa signifiance » (id. : 16), et le traducteur est censé capter la signifiance produite par le(s) rythme(s) du texte et les recréer dans leur spécificité dans une autre langue-culture. Pour ce faire, il ne suffit pas de parler la langue source, il faut « parler texte » : rendre le marqué par le marqué, non-marqué par le non-marqué, la figure par la figure, etc.

Traduire le rythme ne veut pas dire, pour Meschonnic, reproduire forcément le même rythme, mais produire, dans la nouvelle langue-culture, dans le nouveau contexte historique, un rythme qui puisse revêtir les mêmes fonctions et véhiculer les mêmes effets que ceux du texte original. C'est grâce justement à son « mode de fluer », à sa forme dynamique spécifique, à sa signifiance singulière, qu'un texte reste ouvert et toujours réactualisable, donc retraduisible - il est une potentialité langagière et poétique. Par la lecture, les rythmes propres du lecteur se transforment ; en d'autres termes, la littérature a la capacité de transformer les individus, de "transformer les conditions du voir, du sentir, du comprendre », notre manière « $d^{\prime}$ 'être dans le temps et dans l'histoire, [...] d'être avec les autres » (1995a : 142).

\section{Petar Guberina sur le rythme et sa traduction}

Examinons à présent les idées que Petar Guberina a déployées au sujet de la littérature et du style, pour enchaîner ensuite sur sa conception du rythme. Dans sa thèse de doctorat, rédigée à la fin des années 1930, Guberina affirme : « L'homme complet intervient dans l'expression de ses idées. » (1939: 66) ${ }^{4}$ Pour lui, un écrivain est un artiste qui

en dépassant toutes les formes, qui se limitent à ce qui est déjà connu, confirmé par des expériences, trouve dans les valeurs de la langue parlée ce contenu-forme qui fait partie de la vie quotidienne d'un homme adulte, mais qui est inépuisable dans ses variations. (2016 [1967] : 1, III)

Le lecteur, quant à lui, expérimente une œuvre littéraire dans son ensemble ; toute analyse consiste en réalité à analyser notre propre expérience de l'œuvre : «En quelque sorte, nous analysons nous-mêmes, notre monde [...]. C'est pour cela qu'une œuvre est permanente et inépuisable. » (id. : 2, III)

Guberina a conçu le concept de stylographie, ayant pour objectif d'examiner les procédés de style littéraire, les expressions tant affectives que non-affectives dont l'écrivain se sert ( $c f$. Guberina, 1939 : 258). Selon Pavelin-Lešić, il s'agit en grande partie de « retracer les éléments de l'oralité dans l'œuvre littéraire » (2013: 100).

4 La traduction de toutes les citations de Guberina sont faites par nous. Faute de place, nous ne pouvons pas reproduire ici l'original croate. 
En 1967, donc quelques années avant Meschonnic, Guberina s'exprime sur le potentiel esthétique des valeurs de la langue parlée, sur le rythme, ainsi que sur le texte en tant que véritable objet de l'analyse stylographique :

Les mots et les valeurs de la langue parlée représentent également un matériau dont se servira l'artiste, mais cette fois-ci ces éléments seront soumis à la totalité de l'œuvre artistique et se trouveront dans un état potentiel, pouvant se réaliser et se transformer en valeurs esthétiques. (...) Par ailleurs, la stylographie étudiera des formes spécifiques des procédés stylistiques, tels que : le rythme, la symétrie et la dissymétrie, différentes couches (types) de langage; les discours direct et indirect, ainsi que le discours indirect libre, tout cela au service du style; la construction des images. [...] Le critère d'analyse stylographique [...] dépasse les critères grammatical et stylistique. Le point de départ et d'arrivée de l'analyse stylographique est le texte. (2016 [1967] :1, VI)

Tout un chapitre de son ouvrage La stylistique est consacré au rythme. Guberina y anticipe la position de Meschonnic, quand il dit :

À mon avis, le rythme n'est pas une notion désignant un flux monotone ; le rythme n'est pas une notion désignant la répétition des éléments fixes à intervalles identiques ; le rythme n'est pas une notion englobant le décompte des syllabes et l'enchaînement des rimes. Si le rythme l'était, l'art ne serait pas de l'art, car l'art serait alors un ensemble de schémas, d'unités temporelles fixes et de suites de mots ayant le même nombre de syllabes. (id. : 2, I)

Guberina considère que le rythme doit être analysé selon trois fonctions : a) comme la quantité du temps ; y compris la pause en tant que temps actif ; b) comme le développement des périodes ; c) comme la fonction de la représentation des thèmes. Le rythme est pour lui « l'élément principal d'une œuvre littéraire », il est une force qui organise le texte :

Il existe un fil, une force qui crée l'axe même de l'architecture de tous les éléments lexicaux et acoustiques et visuels ; qui construit la ligne de l'expression verbale à partir de cette masse expressive, où le mot en tant que tel n'est qu'une partie de l'édifice expressif. Ce fil, cet axe, qui organise tout et qui, ce faisant, devient la base même de l'expression artistique - c'est le rythme. [...] Observer, donc, le rythme d'une œuvre comme axe de l'expression verbale revient à observer à chaque fois la forme de la pensée artistique, agencée sur la ligne lexicale-musicale-visuelle. [...] le rythme est la partie fondamentale du poème. (ibid.)

Selon Guberina, le rythme est « une construction architectonique et un résultat architectonique de la matière reposant sur la signification multiple et stratifiée des valeurs de la langue parlée et des mots qui nécessairement passent par des périodes temporelles et des segments spatiaux. » (ibid.)

À quel point le rythme est une notion fondamentale dans un texte littéraire, le théoricien croate nous le fait comprendre quand il dit :

Par conséquent, l'étude du rythme dans un texte comprend l'étude de sa totalité expressive [...] Le rythme est donc porteur de l'expression au sens de la pensée artistique, au sens de la suggestivité artistique du texte. (ibid.) 
Le rythme est pour Guberina, tout comme pour Meschonnic, étroitement lié à la parole et à l'oralité, et par là à la réalité de l'auteur, qui est, certes, une réalité subjective ; l'expression de cette réalité ne saurait se limiter à la seule signification :

Le rythme dans les vers et dans la prose s'obtient par la disposition créative des valeurs de la langue parlée, par leur composition dans l'ensemble de l'expression langagière et par la combinaison des sons en un mot articulé. C'est dans la teneur de la langue parlée que le rythme déploie l'expression de base de la pensée artistique. [...] Dans le rythme, c'est la réalité qui parle, celle que l'artiste ressent profondément : il nous transmet sa pensée artistique par le rythme, et nous pénétrons cette réalité par le biais du rythme de l'artiste. Et les mots, articulés, encadrés, se libèrent de leurs contraintes grâce aux valeurs de la langue parlée, lesquelles, au niveau de la qualité artistique, font que ces mots sont plus que la somme des significations selon leur nature lexicale, mais aussi selon leur sonorité. Ainsi le mot devient le son le plus riche en contenu. Et sa signification est plus précise que dans n'importe quel dictionnaire. (id. : 1, III)

Toujours dans le même ouvrage, Petar Guberina ne manque pas de s'exprimer sur l'activité de traduction. Le traducteur est censé analyser tous les éléments de l'expression langagière, y compris les matériaux sonore et lexical, l'intonation, la mimique, le geste, etc. Cette analyse de l'ensemble ne peut pas refléter les lois grammaticales d'une manière mécanique : «Une traduction ne peut pas être segmentée en sons, formes et syntaxes, comme dans les grammaires. » (id. : 2, II) Guberina insiste sur le rôle du contexte qui oriente l'interprétation du texte. La traduction des vers exige que le traducteur porte une attention particulière au langage spécifique du poète, à l'ordre de l'exposition des idées, ainsi qu'au rythme - ce sont toutes des marques de la subjectivité.

Guberina distingue un rythme de base du poème, qui devrait être sauvegardé dans la traduction, et un rythme relatif, qui porte sur une partie du poème, voire sur un seul vers. Il considère que le rythme relatif peut être modifié si l'effet du poème reste préservé. Le traducteur est censé bien traduire le rythme ou les rythmes, spécifique(s) à chaque poète : «Plus il [le rythme] est spécifique, plus il faut lui être fidèle et le rendre aussi bien globalement que dans les détails. » (ibid.)

\section{Quelques remarques conclusives}

Après tout ce que nous venons de dire, nous pouvons constater que Guberina réfléchit sur le rythme et la traduction dans la même veine, mais quelques ans avant Meschonnic. La réflexion de Guberina, ancrée dans la théorie de Charles Bally, anticipe la linguistique textuelle et la stylistique de discours. Certes, l'historicité n'est pas présente dans la réflexion de Guberina - en tant que stylisticien, il est explicitement sur la position synchronique. Par ailleurs, Meschonnic qui poursuit la théorie d'Émile Benveniste dans la direction d'une poétique discursive, pousse sa réflexion plus loin, vers la société, notamment dans ses aspects historiques, éthiques et politiques (idéologiques). 
Néanmoins, il est possible de percevoir quelques convergences importantes dans leurs réflexions respectives. Leur point de départ commun est l'oralité (la Bible pour Meschonnic, la poésie africaine pour Guberina) ; dans leurs considérations, les deux introduisent explicitement le corps en tant qu'instrument produisant la voix, donc le rythme. Pour les deux théoriciens, le texte doit être considéré comme l'unité de base, et le rythme comme l'axe, le principe organisateur du texte littéraire. Les deux insistent sur la continuité discursive et l'interdépendance des éléments discursifs. La question du rythme est pour les deux incontournable dans la traduction de tous les genres littéraires, sans se limiter à la poésie. Enfin, tant Guberina que Meschonnic voient dans la traduction un travail de création artistique lors duquel il est primordial de capter le rythme de l'original en tant que déploiement de la subjectivité dans le texte.

Il ne nous reste qu'à conclure que ces quelques idées portant sur le rythme et sa traduction, nées et approfondies au cours du XXe siècle en Croatie et en France, restent valables aujourd'hui et peuvent servir de repères théoriques précieux pour la pratique traductive littéraire.

\section{Références bibliographiques}

Guberina, Petar (1993). Valeur logique et valeur stylistique des propositions complexes en français et en croate, Zagreb / Mons : Gaj / Didier Erudition/CIPA [thèse soutenue 1939].

Guberina, Petar (2016). Stilistika, Zagreb: stilistika.org, 2016, https://stilistika.org/ petar-guberina-stilistika (22/05/2020) [1 ${ }^{\text {ère }}$ édition 1967];

Meschonnic, Henri (2009). Critique du rythme, Anthropologie historique du langage, Lagrasse: Verdier poche. [1 $1^{\text {èr }}$ édition 1982]

Meschonnic, Henri (1995a). Politique du rythme, politique du sujet, Lagrasse: Verdier.

Meschonnic, Henri (1995b). Traduire ce que les mots ne disent pas, mais ce qu'ils font, in : Meta: journal des traducteurs, 40 (3), pp. 514-517.

Meschonnic, Henri (1999). Poétique du traduire, Lagrasse: Verdier.

Pavelin-Lešić, Bogdanka (2013). L'affectivité au cœur même de la cognition et du langage : Charles Bally et Petar Guberina, in : Synergies Espagne, 6, pp. 93-104. 


\section{Henri Meschonnic, Petar Guberina i ritam u prevođenju}

Tijekom prijevodnog procesa svakom se književnom prevoditelju nužno nameće pitanje ritma, koji sudjeluje u nastanku teksta u njegovoj singularnosti te stvara poetičke učinke. U ovome radu usporedili smo traduktološka razmišljanja o ritmu dvojice velikih teoretičara 20. stoljeća, hrvatskog stilističara Petra Guberine i francuskog poetičara Henrija Meschonnica. U zaključnom smo dijelu, usprkos njihovim različitim teorijskim pozicijama, istaknuli i nekoliko konvergentnih mjesta, vezanih za poimanje teksta, govora (tijela, glasa) i usmenosti, uloge što je ritam u svakom književnom tekstu ima te njegova presudna značaja u prijevodnom procesu. Te su konvergencije po našem mišljenju dragocjeni teorijski orijentiri u današnjoj književnoprijevodnoj praksi.

Ključne riječi: Guberina, Meschonnic, ritam, usmenost, prevođenje 